\title{
Chronic fatigue and psychiatric comorbidities in patients with rheumatic disorders
}

\author{
Luana Andreea Macovei, Irina Dobrin, Vasile Chiriță, \\ Alexandra Burlui, Romeo Petru Dobrin, Elena Rezuș
}

\begin{abstract}
Luana Andreea Macovei - Lecturer, MD, PhD, „Grigore T. Popa” University of Medicine and Pharmacy, Iași, Clinical Rehabilitation Hospital, Iași, Romania

Irina Dobrin - Lecturer, MD, PhD, psychiatrist, „Grigore T. Popa” University of Medicine and Pharmacy, Iași, „Socola” Institute of Psychiatry Iasi, Romania

Vasile Chiriță - Academician, MD, PhD, senior psychiatrist, Socola Institute of Psychiatry, Iași, Romania

Alexandra Burlui - Assistant Lecturer, MD, PhD, „Grigore T. Popa” University of Medicine and Pharmacy, Iași, Clinical Rehabilitation Hospital, Iași, Romania

Romeo Petru Dobrin - Lecturer, MD, PhD, senior psychiatrist, „Grigore T. Popa” University of Medicine and Pharmacy, Iași, Socola Institute of Psychiatry, Iași, Romania

Elena Rezuş - Associate Professor, MD, PhD, „Grigore T. Popa” University of Medicine and Pharmacy, Iași, Clinical Rehabilitation Hospital, Iași, Romania
\end{abstract}

\begin{abstract}
The onset of fatigue has a major impact on the individual and social development of a person, due to its negative effects on health. Chronic fatigue leads to decreased immunity and neuropsychiatric disorders. Chronic fatigue can manifest itself as a neurosis with mental or psychosomatic symptoms, such as ulcer or blood pressure, sleep disturbances, psychoemotional disorders, sensory disturbances and various neurovegetative changes. Fatigue occurs after periods of stress, intense physical activity, lack of sleep or prolonged illness. Chronic fatigue syndrome is found in cases where a few weeks of rest do not improve the condition. The treatment of chronic fatigue is individualized according to the underlying cause of fatigue. By helping patients with rheumatic diseases to adopt healthier and more active lifestyle habits, cognitive behavioural therapy has proved to be effective for reducing fatigue in these cases characterized by intense pain and poor prognosis.
\end{abstract}

KEYWORDS:

Rheumatoid arthritis, systemic lupus erythematosus, ankylosing spondylitis, depression, cognitive behavioral therapy. 


\section{INTRODUCTION}

Fatigue has emotional, psychological and physical manifestations, such as lack of motivation, energy and concentration, weakness, lack of refreshing sleep, increased emotional sensitivity and short-term memory loss. This implies a classification in mental and physical fatigue. Mental fatigue causes decreased physical performance by neural and not by cardiorespiratory or muscular mechanisms $(1,2)$. There are two fatigue syndromes: chronic fatigue syndrome and idiopathic chronic fatigue. Idiopathic chronic fatigue occurs when the fatigue is debilitating and persists for over 6 months, without meeting the diagnostic criteria for chronic fatigue syndrome.

Fatigue is a protective adaptive cortical response expressed as a reversible decrease in working capacity. It is caused not only by expended effort during various activities, but also by underlying pathologies. Except for attention, the psychological aspects of fatigue are rather difficult to assess, unlike the physiological aspects of fatigue which can be accurately measured in cells and muscle fibers.

The neuromuscular system cannot deal with efforts which exceed its functional capabilities. Local fatigue involves muscle fatigue, which is manifested as chemical, metabolic, thermal, mechanical and electrical processes, including depletion of energy reserves in the muscles associated with hypoxia, a deficiency of oxygen supply to the muscle, build up of oxidation products and fatigue toxins in muscles, development of specific nerve fatigue to muscle and heterochronism. Besides muscles, cerebral cortex cells are also exposed to fatigue, whereas the nerves remain unaffected. The motor nerve centers are the first to be affected and muscle contraction is reduced.

Cell fatigue is associated with changes in osmotic pressure, electrical conductivity, ion- exchange reactions and most of all with changes in tissue water. Fatigue leads to the disruption of hydric exchanges, which occurs mostly in the cytoplasm, and damage of the nucleic acid regulating the muscle cell metabolism. Enzymes that control muscle contractions are also affected. Fatigue alters also the structure of the contractile proteins, actin and myosin.

Nervous fatigue can be related to insomnia. Both are stress factors that cause cellular and metabolic disturbances, such as changes in neuromuscular excitability.

Causes of fatigue related to the nature of work include poor organization of work (intense physical effort, prolonged working hours, noise, unfavorable atmosphere, improper lighting). Psychological overload due to sustained attention, monotonous or repetitive work, heavy responsibility and conflicting workplace relationships or professional dissatisfaction. Work capacity is directly related to the health status of the individual, but socioeconomic and family factors can also contribute to fatigue.

Neurotic disorders lead to temporary incapacity for work which poses major socioeconomic challenges. On the other hand, living conditions, social relationships and economic well-being impact mental health.

The search strategy was applied to Pubmed MEDLINE (508 results), Web of Science (78 results), Scopus (203 results), Ebsco Academic Search Complete (27 results), Science Direct (1028 results), Springer Link (5223 results), ProQuest (5795 results) and Wiley Online Library (7952 results). Last search was performed in December, 2018.

The search terms included (fatigue OR exhaustion OR tiredness) AND ((psychiatric disorder) OR (psychiatric illness) OR (mental health) OR (mental illness) OR (mental disorder) OR depression OR anxiety) AND ((rheumatic disorder) OR rheum* OR (rheumatoid arthritis) OR (systemic lupus 


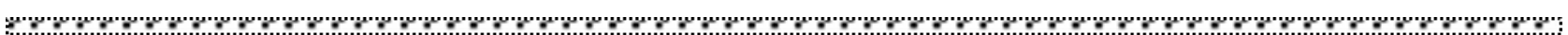

erythematosus) OR (ankylosing spondylitis).

The reference lists of recent reviews on this topic were also investigated.

\section{STUDY SELECTION}

The search result yielded a large and heterogeneous number of items. Duplicates, meeting abstracts, book reviews, editorials and irrelevant articles have been excluded after reading their titles, abstracts, tables and charts. A peer reviewed limit refined our search and only the articles published after 2000 were considered.

\section{DISCUSSION}

Chronic fatigue can be manifested as a neurosis with mental or psychosomatic symptoms that can lead to the appearance of systemic disorders. Chronic fatigue syndrome can cause depression, generalized anxiety, panic and somatization disorders. Sleep disturbances, psycho-emotional disorders, sensory disturbances (cenesthopathy, paresthesia, dizziness, balance disorders, visual disturbances) and various neurovegetative changes.

Active or passive rest is of little or no use for patients with chronic fatigue syndrome, a condition which is defined as a severe tiredness which does not improve with rest. Rest and stress prevention are recommended, but these patients are so agitated, that they cannot relax anymore.

Prolonged stress causes symptoms that can indicate chronic fatigue: dizziness, fainting, severe headache, insomnia, daytime tiredness, difficulty concentrating, memory loss, reasoning errors, increased irritability or intolerance to noise and bright lights, increased heart rate, palpitations, chest pain, fluctuating body temperature, increased blood pressure, muscle and joint pain, numbness, sweating, back pain, spinal sensitization, swallowing difficulty, nausea, gastritis/ulcer, bowel disorders. The severity of fatigue depends mostly on the physical and mental resources of an individual and less on the causing situation. People with normal fatigue are able to recover after adequate rest. If exhaustion is prolonged, mental disorders may occur (3).

When fatigue is present, a psychiatric disorder is 10 times more likely to be diagnosed than chronic fatigue syndrome (4). Psychiatric disorders affect up to two thirds of chronic fatigue syndrome patients. Many patients with fatigue are unwilling to accept a psychiatric diagnosis and those who have received a psychiatric diagnosis might not be able to realistically report their fatigue symptoms, which characterize psychiatric disorders such as depression, bipolar disorder, mood disorders caused by various diseases, posttraumatic stress disorder or anxiety. Depression is the most frequent psychiatric diagnosis made in patients complaining of chronic fatigue, almost $30 \%$ of patients with depression showing this symptom. The symptoms of fatigue may reduce self-care behaviours and leisure activities, which may contribute to depressed mood. Depression and fatigue share many common features (e.g. irritability, feeling overwhelmed, and impaired physical and cognitive functioning, underlying causes such as sleep disturbances, physiological changes or critical life events, weight loss) that can make them difficult to differentiate in clinical practice. Fatigue is present in the early stages of burnout, while the late stages indicate symptoms characteristic to major psychiatric disorders, such as depression and neurasthenia. Chronic fatigue is due to external causes, whereas depression is associated with self-blame and is context-free. Chronic fatigue syndrome is more often associated with frustration and stress, as patients do not feel up to their expectations, while depression is characterized rather by apathy and loss of interest. Asking the patient about loss of 
(1)

appetite, hopelessness, sadness and suicidal thoughts can help diagnose depression. For a differential diagnosis between chronic fatigue syndrome and psychiatric disorders, patients under suspicion are often referred to the psychiatrist (5).

Fatigue is also the chief complain in patients with sleep disorders, such as hypersomnia, insomnia, behaviorally induced insufficient sleep syndrome, circadian rhythm disorders (shift work type), narcolepsy and sleep apnea. In order to differentiate fatigue from sleepiness, the onset time of fatigue and its evolution should be identified during anamnesis and physical examination, having in mind that fatigue is not associated with an increased tendency to fall asleep. Burnout and exhaustion disorder are further related concepts.

Attention-deficit/hyperactivity disorder (ADHD), delirium, Alzheimer disease, personality changes caused by various diseases, substance intoxication (e.g. alcohol and drug abuse), schizophrenia share symptoms with fatigue, i.e. attention deficit, impaired consciousness and memory, decision making problems, inability to concentrate, non-refreshing sleep.

Female gender, emotional instability, prolonged childhood sickness, physical inactivity and overweight, extreme energy during childhood and overactive behavior, traumatic life events are risk factors for fatigue and subsequent psychiatric disorders. Chronic fatigue syndrome is diagnosed after at least 6 months of unexplained fatigue and at least four out of eight somatic or cognitive symptoms, such as musculoskeletal pain, memory and concentration problems, excessive irritability, confusion, difficulty thinking clearly, headache, sleep disturbances, post-exertional discomfort (e.g. flu-like symptoms), sore throats, nausea and lymph node sensitivity. Neurasthenia is characterized by an unspecified duration of fatigue and two associated somatic symptoms. In children, fatigue needs to have been present for at least 3 months.

Due to the fact that fatigue is characterized by mood swings, anxiety, irritability or panic attacks, fatigue can be treated by psychological methods, such as cognitive behavioral therapy, as well as physical exercise, psychotropic drugs, stimulants, antidepressants and thyroid preparations, such as D-amphetamine, methylphenidate, modafinil, armodafinil, selegiline, amantadine, pergolide, bupropion and Lthyroxine. Painkillers and dietary supplements, such as essential fatty acids and magnesium are also use to relieve fatigue symptoms $(6,7)$.

Finding the source of fatigue is important in treatment strategy. If a medical condition is the cause of fatigue, a targeted treatment of the disease would relieve fatigue symptoms (except for persistent or recurrent diseases, such as major depressive disorder or chronic hepatitis C), whereas a patient with fatigue caused by insufficient sleep shows improvement after increased sleep duration. Nevertheless, some medications cause fatigue: antidepressants, antihistamines, antihypertensives, diuretics, sedatives, hypnotics, lithium, beta blockers and steroids. Antidepressants used for the treatment of chronic fatigue syndrome include fluoxetine, phenelzine, sertralin and clomipramine. However, antidepressants are prescribed only for patients with major depression associated with chronic fatigue symptoms, but severe muscle pain and sleeping disorders can also be treated with low doses of tricyclic antidepressants.

Behavioral methods used for fatigue relief include better sleep, a healthy balance diet with at least 2 liters of water per day, regular physical exercise and relaxation techniques, avoiding stress and the use of alcohol, tobacco, recreational drug and excessive 
(1)

caffeine (8). Cognitive behavioral therapy aims at adopting lifestyle habits that may alleviate symptoms, such as avoiding too much rest, with a graded increase in patient's activity. Cognitive behavioural therapy used for fatigue is similar to that used for various rheumatic diseases (7).

The prevalence of fatigue in cancer patients ranges from 37 to $100 \%$, from 40 to $70 \%$ in stroke patients, being of $40 \%$ in patients with inflammatory bowel disease. Fatigue occurs in many underlying medical conditions unrelated to rheumatic diseases, such as congestive heart failure, Addison's disease, anemia, chronic obstructive pulmonary disease, eating disorders, diabetes mellitus, multiple sclerosis, cancer, hypercalcemia, kidney disease, liver disease, hypothyroidism or hyperthyroidism, traumatic brain injuries, neurologic disorders (Parkinson's disease, stroke, multiple sclerosis), long-lasting infection (Epstein-Barr virus and Coxiella burnetii, the human immunodeficiency virus, enteroviruses, bacterial endocarditis, cytomegalovirus, tuberculosis, mononucleosis), muscular dystrophy and old age.

The history taking of the chronic fatigue patients concerns current stressors, occupational, marital and financial problems, head injuries or concussions. Physical examination may provide valuable data on patient's susceptibility to sleep apnea, psychiatric disorders and thyroid disease. Blood tests are performed in order to identify the presence of underlying conditions, such as anemia, diabetes, infection, renal or liver disease. Vitamin D deficiency has also been associated with fatigue and excessive sleepiness.

It is difficult to develop objective assessments of mental fatigue because there are only subjective fatigue measures based on the patient's perception of fatigue. However, mental fatigue assessed by using Functional
Magnetic Resonance Imaging helps track brain activity during cognitive tasks. Imagistic investigations show functional and structural abnormalities in the brains of patients with chronic fatigue syndrome, such as reduced cerebral blood flow and brain volume loss (9).

Twin studies have shown a moderate genetic risk in chronic fatigue syndrome. Obsessivecompulsive personality traits, overactivity, perfectionism, all-or-nothing behavior, the tendency to persevere despite exhaustion are predisposing factors for chronic fatigue syndrome. The estimated prevalence of chronic fatigue syndrome ranges from $0.06 \%$ to $5 \%(10,11)$.

Rheumatic disorders disrupt sleep through pain or discomfort, leading to sleepiness during waking hours. Myalgias, arthralgias, difficulty walking, weakness, inability to continue with daily activities are a common symptom of chronic fatigue syndrome and rheumatic diseases (12-14). The therapy used in patients with rheumatic diseases aims at reversing fatigue effects. Due to the fact that rheumatic diseases are known to worsen over time, these patients are prone to depression $(15,16)$.

Up to $70 \%$ of fibromyalgia cases are associated with chronic fatigue syndrome. Fatigue is present in rheumatoid arthritis, seronegative spondyloarthropathies (psoriatic arthritis, seronegative spondyloarthritis, enteropathic arthritis, ankylosing spondylitis, reactive arthritis) and connective tissue diseases (systemic lupus erythematosus, myositis, vasculitis, Behçet's disease, Sjögren's syndrome). In these instances, fatigue can be caused by sleep problems related to persistent pain, older age, obesity and depressive moods. Sleep fragmentation impairs central pain modulatory processes, worsening pain symptoms and causing stiffness the following day, which leads to furthermore sleep deprivation. Patients with 
(1)

cervical spine involvement and obesity may suffer from obstructive sleep apnea.

Fatigue is correlated with higher levels of Creactive protein, indicating that inflammation favors this symptom. Even if osteoarthritis is generally viewed as a noninflammatory disease and therefore unlikely to cause fatigue, pain is the strongest predictor of fatigue and the sleep of osteoarthritis patients is more shallow than normal. In patients with systemic lupus erythematosus, sleep disturbances are usually caused by depressed mood, pain, prednisone use, and lack of physical exercise $(17,18)$.

Physical disability caused by rheumatic diseases is the cause of physical fatigue, whereas the exhaustion caused by sleep deprivation is referred to as mental fatigue. Drug therapy, aging and depressive moods are also linked to fatigue. Patients try to alleviate fatigue by getting more rest (e.g. diurnal sleep), by using assistive devices and by seeking family and friend companionship. Physical exercise can be used as a method of energizing and improving nocturnal sleep.

Chronic fatigue syndrome can be assessed using the following measuring tools: Chalder Fatigue Questionnaire (an eleven-item scale for physical and mental fatigue), the Fatigue Acceptance Questionnaire (a nine-item scale for lack of acceptance of fatigue symptoms) and Functional Assessment of Chronic Illness Therapy Fatigue Scale - FACIT (a thirteenitem scale for overall fatigue severity) (19).

Fatigue is a common symptom in rheumatoid arthritis patients and it is considered highly relevant for the quality of life. It was estimated that intense fatigue symptoms are reported by up to $80 \%$ of rheumatoid patients, being often associated with disease activity (20).
In rheumatoid arthritis patients, fatigue is significantly reduced after use of the antiTNF inhibitors, such as etanercept and adalimumab, suggesting the involvement of cytokines in the pathogenesis of fatigue, whereas leflunomide and methotrexate prove unable to relieve fatigue.

As a rheumatoid arthritis symptom, fatigue seems to be more intense in the early afternoon. However, fatigue is ameliorated following disease treatment, and it is absent in most patients in remission.

Fatigue is a very common symptom in systemic lupus erythematosus patients and it has been reported by up to $80 \%$ of patients at some time during their illness. Several conditions associated with fatigue are frequently comorbid with systemic lupus erythematosus, including psychiatric disorders, such as depression. Drug therapy with corticosteroids may also worsen sleep quality (21).

Severe fatigue is one of the most frequently reported symptoms of Sjögren's syndrome. The incidence of fatigue in Sjögren's syndrome is high (57\%) and often linked to dysfunction of the autonomic nervous system and frequent co-morbidities (22).

Ankylosing spondylitis patients experience more often fatigue than the general population (23).

Cognitive behavioral therapy may help patients with rheumatic diseases to adopt healthier and more active lifestyle habits. Adaptive Pacing Therapy is also used and patients are advised to adapt demanding physical activities to their individual needs and capabilities, by balancing aspirations and strengths. 


\section{CONCLUSIONS}

Fatigue is present in rheumatic diseases. Sleep disturbances, fatigue, pain, and disability are closely related in the perceived mental and physical well-being of patients with rheumatic diseases. Cognitive-behavioural therapy has proved to be effective for reducing fatigue in rheumatic disease patients. Due to the major impact of fatigue on the quality of life and functional outcomes of patients with rheumatic diseases, fatigue and depressive symptoms should be addressed in rehabilitation settings not only by psychologists, but also, if necessary, by psychiatrists.

\section{ACKNOWLEDGEMENTS AND DISCLOSURES}

The authors state that there are no declared conflicts of interest regarding this paper.

\section{REFERENCES}

1. 1. Stutz P.V., Golani L.K., Witkin J.M.: Animal models of fatigue in major depressive disorder. Physiol Behav 2019; 199: 300-305.

2. Connaughton J., Patman S., Pardoe C.: Are there associations among physical activity, fatigue, sleep quality and pain in people with mental illness? A pilot study. J Psychiatr Ment Health Nurs 2014; 21: 738-745.

3. Nuallaong W. Burnout Symptoms and Cycles of Burnout: The Comparison with Psychiatric Disorders and Aspects of Approaches. In: Bährer-Kohler S, editor, pp. 47-72.

4. Wyller V.B., Eriksen H.R., Malterud K.: Can sustained arousal explain the Chronic Fatigue Syndrome? Behav Brain Funct 2009; 5: 10.

5. Wilson N., Wynter K., Fisher J., et al.: Related but different: distinguishing postpartum depression and fatigue among women seeking help for unsettled infant behaviours. BMC Psychiatry 2018; 18; 309.

6. Harvey S.B., Wessely S., Kuh D., et al.: The relationship between fatigue and psychiatric disorders: evidence for the concept of neurasthenia. J Psychosom Res 2009; 66: 445-454.

7. Van Duyse A., Mariman A., Poppe C., et al.: Chronic fatigue syndrome in the psychiatric practice. Acta Neuropsychiatr 2002;14: 127-133.

8. Ahmed I., Thorpy M. Clinical Assessment of Medical, Neurological, and Psychiatric Conditions Associated with Fatigue. In: Sharafkhaneh A, Hirshkowitz M, editors, pp. 69-91.

9. Wostyn P., De Deyn P.P.: The putative glymphatic signature of chronic fatigue syndrome: A new view on the disease pathogenesis and therapy. Med Hypotheses 2018; 118: 142-145.

10. Herman J.: Psychiatric disorders and fatigue. Sleep Med Clin 2013; 8(2):213-219.

11. Skapinakis P., Lewis G., Meltzer H.: Clarifying the relationship between unexplained chronic fatigue and psychiatric morbidity: results from a community survey in Great Britain. Int Rev Psychiatry 2003; 15: 57-64.

12. Lawn T., Kumar P., Knight B., et al.: Psychiatric misdiagnoses in patients with chronic fatigue syndrome. JRSM Short Rep 2010; 1: 28.

13. Tuithof M., Ten Have M., Beekman A., et al.: The interplay between emotional exhaustion, common mental disorders, functioning and health care use in the working population. J Psychosom Res 2017; 100: 8-14.

14. Kempke S., Van Den Eede F., Schotte C., et al.: Prevalence of DSM-IV personality disorders in patients with chronic fatigue syndrome: a controlled study. Int J Behav Med 2013; 20: 219-228.

15. Jones J.F., Lin J.M., Maloney E.M., et al.: An evaluation of exclusionary medical/psychiatric conditions in the definition of chronic fatigue syndrome. BMC Med 2009; 7: 57.

16. Cook K.F., Jensen S.E., Schalet B.D., et al.: PROMIS measures of pain, fatigue, negative affect, physical function, and social function demonstrated clinical validity across a range of chronic conditions. J Clin Epidemiol 2016; 73: 89102.

17. Lam R.W.: Sleep disturbances and depression: a challenge for antidepressants. Int Clin Psychopharmacol 2006; 21: S25-S29.

18. Buenaver L.F., Smith M.T.: Sleep in rheumatic diseases and other painful conditions. Curr Treat Options Neurol. 2007; 9: 325-336.

19. Power J.D., Badley E.M., French M.R., et al.: Fatigue in osteoarthritis: a qualitative study. BMC Musculoskelet Disord 2008; 9: 63. 

20. Ali S., Matcham F., Irving K., et al.: Fatigue and psychosocial variables in autoimmune rheumatic disease and chronic fatigue syndrome: A cross-sectional comparison. J Psychosom Res 2017; 92: 1-8.

21. Chang S., Seow E., Koh S.H.D., et al.: Treatment preferences and help-seeking behaviors for sleep problems among psychiatric outpatients. Gen Hosp Psychiatry 2018; 51; 112-117.

22. Staud R.: Peripheral and central mechanisms of fatigue in inflammatory and noninflammatory rheumatic diseases. Curr Rheumatol Rep 2012; 14(6):539-548.

23. Druce K.L., Cordingley L., Short V., et al.: Quality of life, sleep and rheumatoid arthritis (QUASAR): a protocol for a prospective UK mHealth study to investigate the relationship between sleep and quality of life in adults with rheumatoid arthritis. BMJ Open 2018; 8: e018752.

\section{Correspondence:}

Luana Andreea Macovei,

Lecturer, MD, PhD, „Grigore T. Popa” University of Medicine and Pharmacy, Iași, Clinical Rehabilitation Hospital, Iași, Romania, luanam77@yahoo.com

Submission: 10 may 2019

Acceptance: 29 july 2019 